BMJ

Open

Gastroenterology

\title{
Assessment of Helicobacter pylori status by examination of gastric mucosal patterns: diagnostic accuracy of white-light endoscopy and narrow- band imaging
}

\author{
Ben Glover (10 , Julian Teare, Nisha Patel
}

To cite: Glover B, Teare J, Patel N. Assessment of Helicobacter pylori status by examination of gastric mucosal patterns: diagnostic accuracy of white-light endoscopy and narrow-band imaging. BMJ Open Gastro 2021;8:e000608. doi:10.1136/ bmjgast-2021-000608

- Additional supplemental material is published online only. To view, please visit the journal online (http://dx.doi. org/10.1136/bmjgast-2021 000608).

Received 6 April 2021 Accepted 4 July 2021

\section{Check for updates}

C) Author(s) (or their employer(s)) 2021. Re-use permitted under CC BY-NC. No commercial re-use. See rights and permissions. Published by BMJ.

Surgery and Cancer, Imperial College London, London, UK

Correspondence to Dr Ben Glover;

bglover@ic.ac.uk

\section{ABSTRACT}

Objectives Helicobacter pylori infection is a common cause of chronic gastritis worldwide and an established risk factor for developing gastric malignancy. The endoscopic appearances predicting $H$. pylori status are an ongoing area of research, as are their diagnostic accuracies. This study aimed to establish the diagnostic accuracy of several mucosal features predictive of $H$. pylori negative status and formulate a simple prediction model for use at the time of endoscopy.

Design Patients undergoing high-definition upper gastrointestinal (Gl) endoscopy without magnification were recruited prospectively. During the endoscopy, the presence or absence of specific endoscopic findings was noted. Sydney protocol biopsies were used as the diagnostic reference standard, and urease test if taken. The results informed a logistic regression model used to produce a simple diagnostic approach. This model was subsequently validated using a further cohort of 30 patients.

Results 153 patients were recruited and completed the study protocol. The prevalence of active $H$. pylori infection was $18.3 \%(28 / 153)$. The overall diagnostic accuracy of the simple prediction model was $80.0 \%$, and $100 \%$ of patients with active H. pylori infection were correctly classified. The presence of regular arrangement of collecting venules (RAC) showed a positive predictive value for $H$. pylori naïve status of $90.7 \%$, rising to $93.6 \%$ for patients under the age of 60 .

Conclusion A simple endoscopic model may be accurate for predicting $H$. pylori status of a patient, and the need for biopsy-based tests. The presence of RAC in the stomach is an accurate predictor of $H$. pylori negative status, particularly in patients under the age of 60 .

Trial registration number The study was registered with ClinicalTrials.gov, No. NCT02385045.

\section{INTRODUCTION}

Helicobacter pylori infection is a major cause of gastritis worldwide and is well established as a causative agent in gastric atrophy, intestinal metaplasia and dysplasia, culminating in the development of gastric adenocarcinoma. ${ }^{1}$
Summary

What is already known about this subject?

- The endoscopic appearance of a Helicobacter pylori naiive stomach includes the presence of regular arrangement of collecting venules (RAC) within the distal body. Other features of the $\mathrm{H}$. pylori infected or eradicated stomach have been identified, but these have not been studied as diagnostic indicators to predict $H$. pylori status.

What are the new findings?

- Presence of the RAC in the distal stomach has been prospectively confirmed as a strong predictor of $H$. pylori negative status. Findings of diffuse redness and mucosal oedema have strong predictive value for active $H$. pylori infection. The finding of map-like redness is a strong predictor of previous $H$. pylori eradication. When applied in a simple algorithmic fashion, $80 \%$ of patients can have $H$. pylori status predicted correctly.

How might it impact on clinical practice in the foreseeable future?

- The use of an objective classification system may guide decision making at the time of endoscopy, including further diagnostic tests for $\mathrm{H}$. pylori, avoiding biopsy in negative patients and considering more extensive examination or biopsy in patients at increased risk of gastric cancer.

Detection of $H$. pylori infection and subsequent eradication therapy has been shown to reduce symptoms of dyspepsia, and to stabilise or reduce the risk of developing gastric cancer. $^{23}$

Several techniques exist for diagnosing H. pylori infection, and include non-invasive methods such as urea breath testing, faecal antigen testing and serological tests. Tissue samples may also be obtained during endoscopy and can be evaluated by microscopy, culture or rapid urease test. ${ }^{4}$ In recent years, 

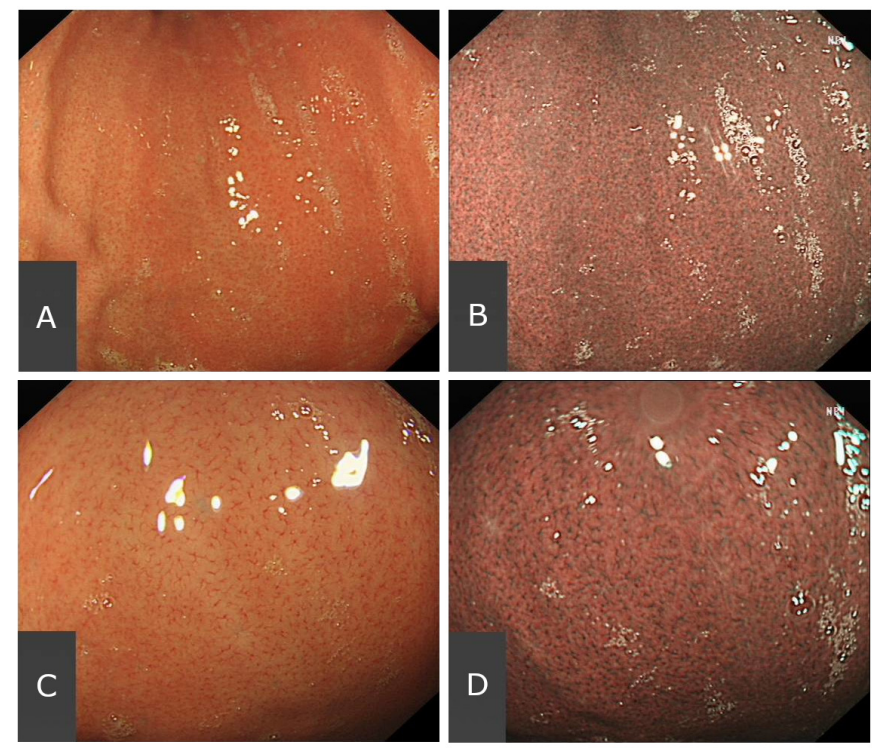

Figure 1 The endoscopic appearance of the RAC in the distal stomach, in a Helicobacter pylori naïve patient. Examined at medium distance without magnification under white-light endoscopy (WLE) (A) and narrow-band imaging (B), and on closer inspection without magnification under WLE (C) and NBI (D). NBI, narrow-band imaging.

the widespread adoption of high-resolution endoscopy platforms has dramatically improved the level of mucosal detail visible to the endoscopist, and advanced imaging modalities such as narrow-band imaging (NBI) can improve visual contrast to better examine fine mucosal features. ${ }^{5}$

It is recognised that $H$. pylori naïve, infected or eradicated states, may be associated with distinct mucosal appearances, and the clinical utility of identifying these patterns is an area of ongoing study. ${ }^{6}$ With adequate accuracy, it could be appropriate to either initiate eradication therapy for suspected $H$. pylori, or identify patients at higher risk of long-term complications, that is, those with current or previous $H$. pylori infection.

A significant area of interest is the presence of a 'regular arrangement of collecting venules' (RAC), first described by Yagi et al, under magnifying and standard endoscopy. ${ }^{7}$ This is seen as numerous dots, or starfish-like points evenly distributed in the gastric body, as shown in figure 1. Further study of the RAC has suggested that when present, the RAC has a negative predictive value (NPV) of $>90 \%$ for $H$. pylori negative status. ${ }^{8-10}$ This may approach $100 \%$ when the stomach is examined with image enhancement, such as NBI. ${ }^{1-13}$ Different types of abnormal RAC pattern have been described ${ }^{14}$; this study has attempted to simplify the diagnostic process by specifying only the presence or absence of the normal RAC, when examined without magnification using both white light and NBI.

However, the applicability of the RAC for diagnosis of an $H$. pylori naïve state may not be universal. Alaboudy et $a l^{15}$ demonstrated that the presence of RAC decreases with age, particularly for patients over the age of 60 .

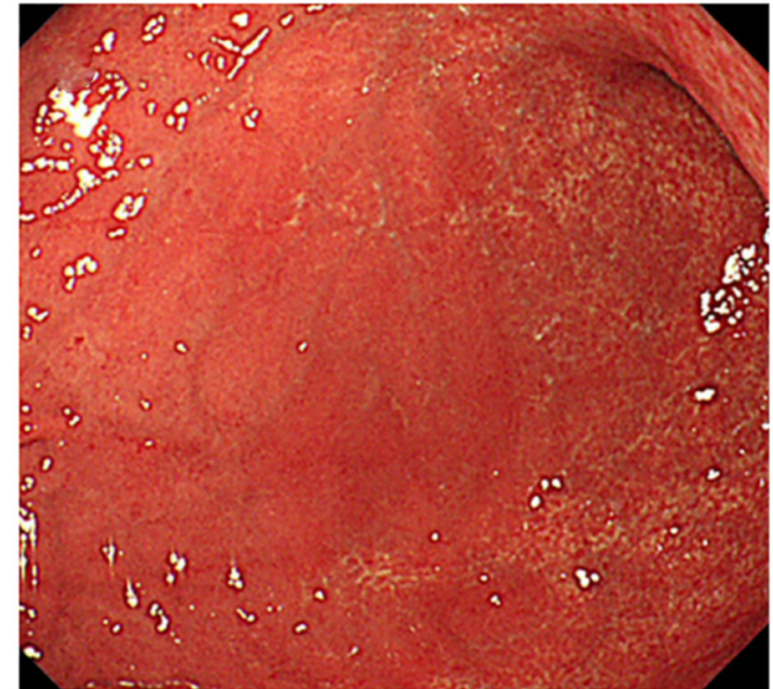

Figure 2 The endoscopic appearances of diffuse redness, in a Helicobacter pylori infected patient. ${ }^{32}$

The prevalence of $H$. pylori infection also varies widely worldwide, from as low as $24.4 \%$ in Oceania, to $70.1 \%$ in Africa. Prevalence in Northern Europe varies from $22.1 \%$ in Denmark, to $82.5 \%$ in Estonia. ${ }^{16}$ In addition, a large proportion of the studies examining RAC are performed in Asian populations by expert endoscopists, and relatively few in Western populations. ${ }^{17} 18$ The use of imageenhanced endoscopy such as NBI may also be of benefit in improving the contrast and delineation of gastric mucosal features and visualising the RAC, although requires further validation. ${ }^{19} 20$

Positive predictive findings for active $H$. pylori infection include diffuse redness, (figure 2) mucosal oedema, (figure 3) antral nodularity, enlarged gastric folds and sticky mucus, all of which may suggest a diagnosis of H. pylori infection. ${ }^{10} 13$ 21-23 The significance of gastric erosions and haem flecks is less clear, with some studies reporting that gastric erosions are a predictor of $H$. pylori naïve status; other reports suggest that presence of gastric erosions may predict active $H$. pylori infection. ${ }^{624-26}$

It is also important to recognise patients with previously eradicated $H$. pylori infection, both to avoid unnecessary treatment and to guide decisions regarding biopsy for assessment of gastric atrophy or intestinal metaplasia. ${ }^{27}$
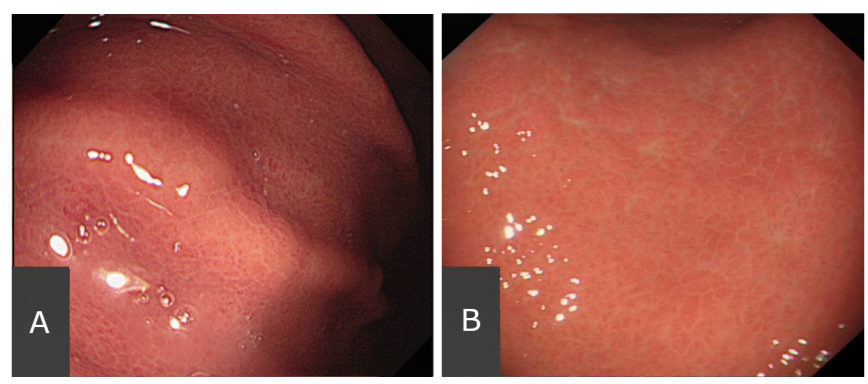

Figure 3 The endoscopic appearances of mucosal oedema, in a Helicobacter pylori infected patient. (A) The gastric body ${ }^{34}$ and $(\mathrm{B})$ under closer inspection near the antrum. 


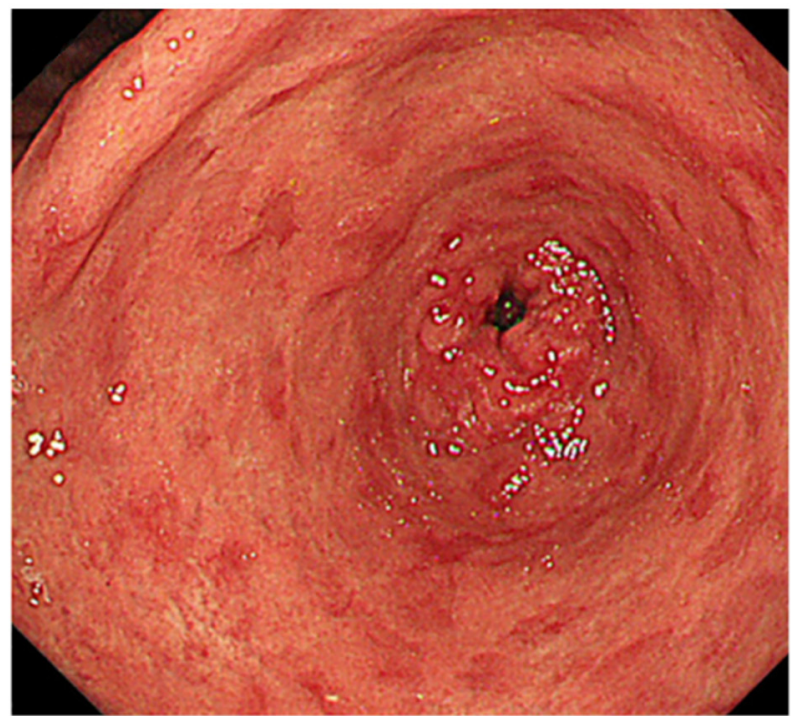

Figure 4 The endoscopic appearances of map-like redness, in a patient with previous Helicobacter pylori eradication. $^{32}$

Previous studies have identified a mucosal pattern of 'map-like redness' (figure 4) as being predictive of $H$. pylori eradicated status or gastric metaplasia. ${ }^{28}$ It has also been shown that some changes can regress following $H$. pylori eradication, including diffuse redness and antral nodularity. ${ }^{29}$

This study attempts to evaluate the diagnostic accuracy characteristics of defined mucosal features as predictors of $H$. pylori status in a European population, and to provide early prospective validation of a simple endoscopic classification system.

\section{METHODS}

\section{Recruitment}

We performed a retrospective and prospective validation of the diagnostic performance of endoscopic findings reported in real-time in the upper GI tract, using conventional white-light endoscopy (WLE) and NBI. After recruitment of an initial cohort of patients we carried out multinomial logistic regression modelling to identify the predictive features associated with $H$. pylori naive, infected and eradicated states. The results of the modelling informed the development of a predictive classification system, which was validated prospectively in the second cohort of patients.

Patients were recruited after informed consent, as a convenience series from January 2018 to May 2020. All patients over the age of 18 attending for diagnostic gastroscopy due to symptoms of abdominal pain or dyspepsia were considered for recruitment. On presentation to the endoscopy department, the patient history and records were screened for suitability, and eligible patients were identified. Prior to the procedure, patients were interviewed and medical records were checked for history of PPI use and history of $H$. pylori diagnosis and treatment.
Exclusion criteria included patients with previous gastric surgery or liver disease, contraindications to biopsy, acute GI bleeding and with known malignancy of the GI tract. Patients were also excluded if they were unable or unwilling to give informed consent, or if consent was withdrawn after the procedure.

\section{Gastroscopy protocol}

Patients fasted overnight for procedures scheduled in the morning, and for 6 hours for afternoon procedures. Procedures were completed using either pharyngeal anaesthesia (Xylocaine spray) or sedation (midazolam \pm fentanyl) or both, according to patient and clinician preference.

Gastroscopies were performed using HD endoscopes (GIF-H290, Olympus) without magnification. The procedures were performed by six experienced endoscopists (>1000 procedures) following familiarisation with the gastroscopy protocol, and data were recorded by members of the research team present during the gastroscopy. Following a standard examination of the duodenum and stomach by the endoscopist, the antrum, incisura and fundus of the stomach were carefully examined under white light with good insufflation of the stomach, and adequate washing of the mucosa if required. The presence or absence of RAC in the lower part of the stomach, diffuse redness, mucosal oedema, map-like redness, erosions, haem flecks, oesophagitis, antral nodularity and gastric or duodenal ulceration, were recorded in real time. These features are associated with $H$. pylori infection, as well as showing regression after $H$. pylori eradication. ${ }^{29}$ The NBI mode of the gastroscope was then activated, and careful examination of the antrum, incisura and fundus was repeated, for assessment of the same characteristics. Biopsies were then taken according to the updated Sydney Protocol (lesser and greater curvatures at the antrum and body, and at the incisura angularis). The gastroscopy was then completed including any other biopsies (including urease test if clinically indicated) at the discretion of the endoscopist.

\section{Data recording}

Patient demographics and baseline characteristics were recorded including age, sex, ethnicity and the experience level of the endoscopist performing the procedure. All assessments of mucosal features were recorded immediately following the end of the procedure and blinded to the results of a urease test (if taken). The mucosal surface patterns were classified as normal or abnormal based on the appearances described by Yoshii et al and Inui et al. ${ }^{28} 30$

$H$. pylori status was determined using the mucosal biopsies obtained according to the updated Sydney protocol; these were labelled according to their anatomical location and analysed by an expert GI histopathologist. Giemsa staining was performed for all patients. In addition, if a urease test was performed during the gastroscopy (Biohit H pylori UFT300), the results of this were recorded, and 
Table 1 Patient characteristics and Helicobacter pylori prevalence

\begin{tabular}{|c|c|c|c|c|c|}
\hline $\begin{array}{l}\text { Patient } \\
\text { characteristics }\end{array}$ & & & H. pylori naïve & $\begin{array}{l}\text { H. pylori active } \\
\text { infection }\end{array}$ & $\begin{array}{l}\text { H. pylori } \\
\text { eradicated }\end{array}$ \\
\hline \multirow[t]{3}{*}{ Age } & All patients & $54.23 \pm 16.80(19-90)$ & $111(72.5 \%)$ & $28(18.3 \%)$ & $14(9.2 \%)$ \\
\hline & $<50$ & $\mathrm{n}=60(39.2 \%)$ & 44 (73.3\%) & $13(21.7 \%)$ & $3(5.0 \%)$ \\
\hline & $\geq 50$ & $n=93(60.8 \%)$ & 67 (72.0\%) & $15(16.1 \%)$ & $11(11.8 \%)$ \\
\hline \multirow[t]{2}{*}{ Gender } & Male & $\mathrm{n}=66(43.1 \%)$ & $46(69.7 \%)$ & $13(19.7 \%)$ & 7 (10.6\%) \\
\hline & Female & $\mathrm{n}=87(56.9 \%)$ & $65(74.7 \%)$ & $15(17.2 \%)$ & 7 (8.0\%) \\
\hline \multirow[t]{5}{*}{ Ethnicity } & European & $90(58.8 \%)$ & 71 (46.4\%) & $11(7.2 \%)$ & $8(5.2 \%)$ \\
\hline & South Asian & $17(11.1 \%)$ & $13(8.5 \%)$ & 4 (2.6\%) & $0(0.0 \%)$ \\
\hline & East Asian & $3(2.0 \%)$ & $3(2.0 \%)$ & $0(0.0 \%)$ & $0(0.0 \%)$ \\
\hline & Afro-Caribbean & $14(9.2 \%)$ & $9(5.9 \%)$ & $2(1.3 \%)$ & $3(2.0 \%)$ \\
\hline & Middle Eastern & 29 (19.0\%) & 15 (9.8\%) & $11(7.2 \%)$ & $3(2.0 \%)$ \\
\hline
\end{tabular}

a patient was classified as $H$. pylori positive if either one or both tests demonstrated the presence of $H$. pylori.

\section{Statistical analysis}

Sample size calculations were performed according to Buderers method, with assumptions that the RAC would have sensitivity $90 \%$ and specificity $90 \%$ for an $H$. pylori naïve status. ${ }^{31}$ A prevalence of $35 \%$ was estimated, based on recent demographic meta-analysis for Northern Europe. ${ }^{16}$ A sample size of 120 patients was calculated to be required.

We present the sensitivity, specificity, positive and NPVs and accuracy of RAC and other mucosal features, with $95 \%$ CIs. All statistical analyses and modelling were performed in IBM SPSS V.25.

The primary outcomes were the accuracy of the RAC and of the other identified mucosal features, for prediction of the H. pylori status. The secondary outcome was

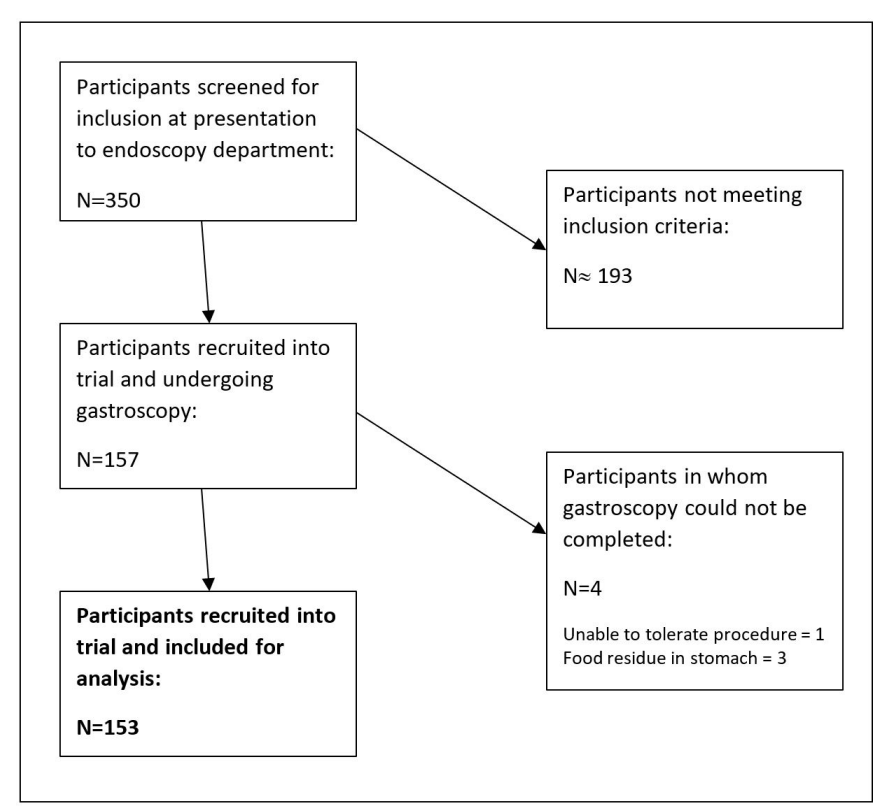

Figure 5 Participant flow diagram. the accuracy of a simple endoscopic system for classifying patients $H$. pylori status.

\section{RESULTS}

\section{Study participants and baseline characteristics}

Approximately 350 patients were screened for inclusion in the study, and 157 patients were recruited; the remainder did not meet inclusion criteria (due to, eg, the procedural indication such as anaemia or dysphagia, previous gastric surgery or liver disease. A further four patients underwent gastroscopy but were subsequently withdrawn from the study due to food residue within the stomach or otherwise incomplete procedure. One patient did not have the biopsy protocol performed; they did, however, have a positive urease test and were therefore included in analysis.

A total of 153 patients were included for analysis; the age range was from 19 to 90 years with a mean age of 54.2 years $( \pm 16.8)$. The participant flow diagram is presented in figure 5 .

\section{H. pylori prevalence}

Using the patient history, histological presence of Helicobacter, or positive urease test, 111 of the 153 patients were $H$. pylori naïve $(72.5 \%), 28$ had active $H$. pylori infection $(18.3 \%)$ and 14 were $H$. pylori eradicated. $32 \%$ of patients underwent urease test, of which 7 were positive; all of these patients also had evidence of $\mathrm{H}$. pylori on histopathological analysis. There were no significant differences in prevalence by age or gender, although the highest prevalence of infection $(11 / 29,37.9 \%)$ was seen in patients from a Middle-Eastern background. Patient characteristics and $H$. pylori prevalence are reported in table 1.

\section{Diagnostic performance of the RAC}

To date, the most extensively studied feature of the stomach for prediction of $H$. pylori status is the RAC (figure 1). Using WLE, the RAC was identified in 108/153 $(70.6 \%)$ of this cohort of patients. All patients with RAC identified under WLE, also showed RAC under NBI. 


\begin{tabular}{|c|c|c|c|c|c|c|}
\hline & $\mathbf{N}$ & $\begin{array}{l}\text { Sensitivity \% } \\
(95 \% \mathrm{Cl})\end{array}$ & $\begin{array}{l}\text { Specificity \% } \\
(95 \% \mathrm{Cl})\end{array}$ & $\begin{array}{l}\text { Positive predictive } \\
\text { value } \%(95 \% \mathrm{Cl})\end{array}$ & $\begin{array}{l}\text { Negative Predictive } \\
\text { Value } \%(95 \% \mathrm{Cl})\end{array}$ & $\begin{array}{l}\text { Accuracy \% } \\
(95 \% \mathrm{Cl})\end{array}$ \\
\hline RAC (all patients) & 153 & 78.4 (70.2 to 85.3$)$ & 64.3 (44.1 to 81.4$)$ & 90.7 (85.5 to 94.2$)$ & 40.0 (30.2 to 50.7$)$ & 75.8 (68.2 to 82.4 ) \\
\hline $\begin{array}{l}\text { RAC (patients } \\
\text { aged <50 years) }\end{array}$ & 60 & 85.1 (71.7 to 93.8$)$ & 76.9 (46.2 to 95.0$)$ & 93.0 (83.1 to 97.3$)$ & 58.8 (40.4 to 75.1$)$ & 83.3 (71.5 to 91.7$)$ \\
\hline $\begin{array}{l}\text { RAC (patients } \\
\text { aged <55 years) }\end{array}$ & 74 & 84.8 (73.0 to 92.8$)$ & 73.3 (44.9 to 92.2 ) & 92.6 (84.4 to 96.7$)$ & 55.0 (38.4 to 70.6$)$ & 82.4 (71.8 to 90.3$)$ \\
\hline $\begin{array}{l}\text { RAC (patients } \\
\text { aged <60 years) }\end{array}$ & 86 & 82.9 (72.0 to 90.8$)$ & 75.0 (47.6 to 92.7 ) & 93.6 (86.0 to 97.2 ) & 50.0 (35.7 to 64.3 ) & 81.4 (71.6 to 89.0$)$ \\
\hline
\end{tabular}

Examined using combined WLE and NBI. Results are presented for different age cohorts.

$\mathrm{NBI}$, negative predictive value; RAC, regular arrangement of collecting venules; WLE, white-light endoscopy.

NBI examination allowed identification of the RAC in a further four patients, all of whom were $H$. pylori negative.

The presence of the RAC in the lower part of the lesser curvature of the stomach has been shown in many previous studies to be a strong predictor of an $H$. pylorinegative status, and our results agreed with this, showing a diagnostic OR of $6.53(95 \%$ CI 2.70 to $15.80, \mathrm{p}<0.001)$ for $H$. pylori negative status (either naive or eradicated) in the presence of RAC.

In $78.4 \%$ of $H$. pylori negative individuals, the RAC was present (under either WLE, NBI or both). By contrast, the RAC was only present in $35.7 \%$ of $\mathrm{H}$. pylori positive patients. Diagnostic performance measures are shown in table 2 for the presence of RAC under combined WLE and IEE examination, as well as for different cut-off values of age, as it has previously been demonstrated that the RAC becomes less visible with increasing age. ${ }^{15}$ For patients below the age of 55 , the presence of RAC carries an NPV of $93.2 \%(95 \%$ CI $83 \% .1$ to $97.3 \%$ ) for active $H$. pylori infection.

\section{Regression modelling of significant endoscopic findings}

A multinomial logistic regression model was employed to evaluate the predictive capabilities of each endoscopic sign being studied (RAC, diffuse redness, mucosal oedema, map-like redness, erosions, ulceration, haem flecks and oesophagitis). For the first cohort of 123 patients, the presence or absence of each feature was recorded during endoscopy, as was any history of $H$. pylori infection or treatment, and the final histological diagnosis. Patients were stratified into 'naïve', 'active infection' or 'eradicated' groups based on their history and the results of biopsy-based tests taken at endoscopy. Results of the endoscopic and histological findings from the patient cohort are presented in table 3 .

A predictive model including all the endoscopic features demonstrated a reasonable goodness-of-fit (multinomial logistic regression modelling, SPSS V.25) with a Pearson $\chi^{2}$ statistic of 16.48 (Sig. 1.00). The processing summary of the final model is presented in online supplemental data. The overall predictive accuracy was $81.3 \%$. Adding the patient age as a covariate did not increase the predictive accuracy of the model, but when the model was applied only to patients under the age of 55 , the expected accuracy rose to $86.4 \%$.
We attempted to produce a predictive model that would preserve accuracy without requiring the endoscopist to record a large number of endoscopic findings; a two-stage predictive model taking previous history into account was therefore developed, after the approach of Gupta $e t a l^{27}$ but requiring fewer endoscopic signs to be assessed. The selected features were presence of RAC, diffuse redness, mucosal oedema and map-like redness (as a marker of atrophy), as these features showed the greatest positive or NPVs for H. pylori status (figures 3-5). Regression modelling suggested a preserved goodness-of-fit, with Pearson $\chi^{2}$ of 21.8 (Sig. 0.005), when including only these findings. The algorithmic approach to the diagnostic prediction is presented in figure 6. Patients in whom none of the included mucosal features could be identified, were classified as 'naive' or 'eradicated' based on any previous history of $H$. pylori infection.

\section{Prospective validation of the diagnostic prediction model}

A further 30 patients were recruited following the development of the diagnostic predictive model. These patients underwent gastroscopy as per the standard protocol. As previously, endoscopists were informed of any history of $H$. pylori infection, but were blinded to results of recent pre-test stool or serological tests for $H$. pylori, and to the results of urease tests taken during endoscopy

Endoscopists were required to state whether each sign in the predictive model was present, and to predict the $H$. pylori status of each patient based on the algorithmic approach detailed previously.

An overall diagnostic accuracy of $80.0 \%$ was demonstrated, the results are presented in table 4 . Six patients had active $H$. pylori infection; all of these were classified correctly. Seventeen patients were $H$. pylori naïve, of which 12 were classified correctly, and the remainder ( 5 patients) were classified incorrectly as having active infection. Three patients were classified as 'uncertain' based on the absence of all studied endoscopic findings but were classified correctly when taking previous history of $H$. pylori into account. No patients with active infection were classified as 'naive' or 'eradicated'. 
Table 3 Frequency of mucosal appearances and histological diagnosis

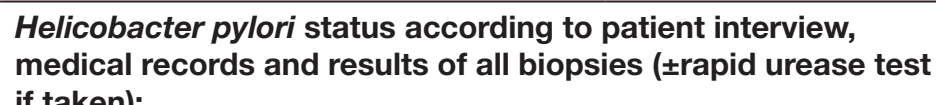
if taken):

\begin{tabular}{|c|c|c|c|}
\hline & \\
\hline & $\begin{array}{l}\text { H. pylori naïve } \\
(\mathrm{n}=111)\end{array}$ & $\begin{array}{l}\text { H. pylori infected } \\
(\mathrm{n}=28)\end{array}$ & $\begin{array}{l}\text { H. pylori eradicated } \\
(n=14)\end{array}$ \\
\hline \multicolumn{4}{|c|}{ Prevalence of endoscopic findings: } \\
\hline Diffuse redness & $27(28.8 \%)$ & $13(46.4 \%)$ & $1(7.1 \%)$ \\
\hline Mucosal oedema & $9(8.1 \%)$ & $7(25.0 \%)$ & $0(0 \%)$ \\
\hline Erosions & $15(13.5 \%)$ & $4(14.3 \%)$ & $1(7.1 \%)$ \\
\hline Gastro-duodenal ulceration & $3(2.7 \%)$ & $4(14.3 \%)$ & $1(7.1 \%)$ \\
\hline Haem flecks & $10(9.0 \%)$ & $0(0 \%)$ & $0(0 \%)$ \\
\hline Oesophagitis & $1(0.9 \%)$ & $1(3.6 \%)$ & $0(0 \%)$ \\
\hline Acute gastritis & $7(6.3 \%)$ & $20(71.4 \%)$ & $0(0 \%)$ \\
\hline Chronic gastritis & $37(33.3 \%)$ & $17(60.7 \%)$ & $7(50.0 \%)$ \\
\hline Gastric atrophy & $2(1.8 \%)$ & $0(0 \%)$ & $4(7.1 \%)$ \\
\hline Gastric metaplasia & $4(3.6 \%)$ & $2(7.1 \%)$ & $4(28.6 \%)$ \\
\hline Helicobacter-like organisms & $0(0 \%)$ & $28(100 \%)$ & $0(0 \%)$ \\
\hline
\end{tabular}

NB: Figures do not sum to $100 \%$ as findings may coexist in patients.

RAC, regular arrangement of collecting venules.

\section{DISCUSSION}

Changes in the endoscopic appearance of the stomach in relation to $H$. pylori infection are an ongoing area of research, and several characteristic features have been identified to predict $H$. pylori naive, positive and eradicated status.
A significant feature of the $H$. pylori naïve stomach is the presence of RAC. Our study supports the previous observations that the presence of this pattern is the strongest single predictor for $H$. pylori naïve status ${ }^{28}$ and therefore careful examination using WLE could be considered as part of a diagnostic approach to 'rule out' $H$. pylori

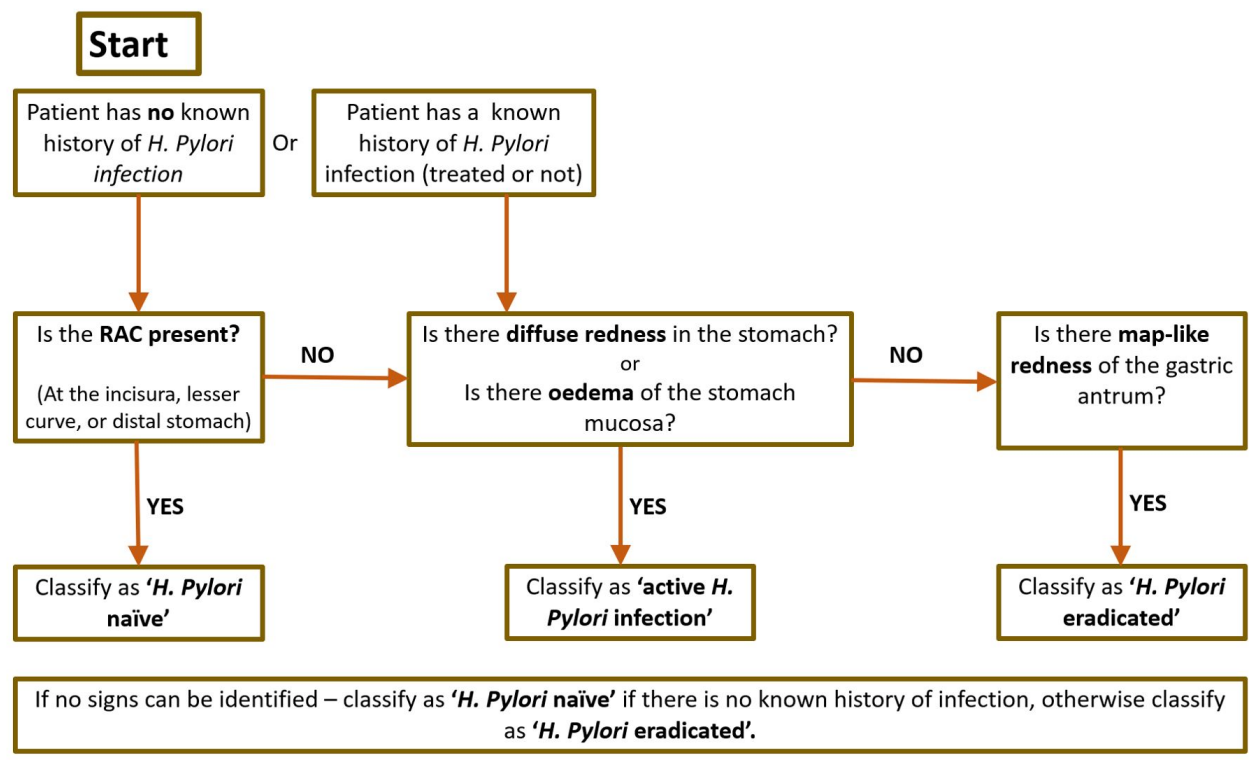

Figure 6 The diagnostic algorithm used for the second cohort of patients to predict classification of Helicobacter pylori status. RAC, regular arrangement of collecting venules . 
Table 4 Results of prospective validation of the diagnostic algorithm

True status based on history, urease test and histology

\begin{tabular}{|c|c|c|c|c|c|}
\hline & & & & & \\
\hline & & Naive & Active infection & Eradicated & Total \\
\hline \multirow{3}{*}{$\begin{array}{l}\text { Predicted Helicobacter pylori status according } \\
\text { to the diagnostic algorithm }\end{array}$} & Naïve & 12 & 0 & 0 & 12 \\
\hline & Active Infection & 5 & 6 & 0 & 11 \\
\hline & Total & 17 & 6 & 7 & 30 \\
\hline
\end{tabular}

infection. Training in the recognition of the RAC would be of benefit in Western endoscopic practice. It is also important to consider that histopathological assessment, as with all biopsy-based techniques, may not diagnose all cases of $H$. pylori if organisms are not present within the biopsy sample area.

Previous similar studies have reported test sensitivity as high as $100 \%$ for $H$. pylori when RAC is present; that is, no patients with RAC present were $H$. pylori positive. Interestingly, our study has found that a significant proportion of $H$. pylori positive patients $(10 / 23)$ were found to have RAC present within the stomach. The reasons for this are unclear; possibilities include that $H$. pylori infection does not always lead to RAC loss, that RAC loss is not immediate or complete, or that RAC was misidentified by the endoscopists in those patients.

We have attempted to produce a diagnostic model to aid in the endoscopic prediction or classification of $H$. pylori status. This model has been validated in a small initial cohort of patients and demonstrated accuracy of $80 \%$. Importantly, no cases of active $H$. pylori infection were classified as naive or eradicated by this method.

When designing this model, it was important to avoid overburdening an endoscopist with a large number of signs and features to identify. Other groups have demonstrated high diagnostic accuracy $(88.6 \%-93.4 \%)$ for an algorithmic approach using history and endoscopic findings, though this was with a machine-learnt model using up to 16 different findings per patient. ${ }^{28}$ The endoscopic features in this approach differ slightly from those in the Kyoto classification, in part due to removing the feature of antral nodularity, which was encountered uncommonly in this population, and also by removing the differentiation between intestinal metaplasia and map-like redness, which are likely to show similar histological findings. ${ }^{32}$

We have proposed a simpler approach which we believe will be more applicable in routine practice. This may aid endoscopists as a screening tool for $H$. pylori naïve patients, and as a decision-making aid for patients who may require further investigation for $H$. pylori or its sequelae, such as atrophic gastritis and metaplasia.

This study has some limitations. The prevalence of $H$. pylori infection was substantially lower $(18.3 \%)$ than the $35 \%$ anticipated based on population studies. This is lower than most globally reported populations and may influence the generalisability of our results. The selection criteria may also limit the wider applicability; this study included only patients with symptoms of dyspepsia or abdominal pain and therefore will not include patients with asymptomatic $H$. pylori infection who have attended for endoscopy for an unrelated reason (such as anaemia or dysphagia).

Similarly, the rates of histologically proven gastric atrophy in $H$. pylori negative patients were low, with only two patients in the $H$. pylori naïve group $(2 / 111)$ and four patients in the $H$. Pylori eradicated group (4/14/) showing histological findings. The reason for this is not clear, although could have important implications for external validity if the rates of gastric atrophy in our cohort differ significantly from those in the wider population.

We also do not know the rates of spontaneous eradication of $H$ pylori; five patients in the initial cohort had no history of infection but did show signs of map-like redness, although no evidence of $H$. pylori was present on biopsy. Additionally, this was a small single-centre study including endoscopists who were motivated and interested to take part. We attempted to assess intraoperator variability but this proved impossible due to the lower image quality of saved files, compared with realtime high-resolution endoscopic assessment. This is an important factor, as features such as mucosal oedema are highly subjective and interoperator and intraoperator agreement should be quantified for these.

If extrapolated to a larger study, it may be appropriate to examine inter-operator variability and the learning curve for applying the proposed algorithm. Furthermore, the prediction algorithm does not consider confounding factors, as each endoscopic feature included is assessed individually, and treated with equal weight. A further limitation is that few participants underwent $H$. pylori stool antigen or urea breath test, and it is understood that biopsy-based diagnosis may carry a higher false-negative rate than these methods. These, however, were not available as patients were recruited as part of their normal routine healthcare.

Another important question is of the effect of PPI treatment on the gastric mucosal appearances. We have included PPI-naïve and PPI-exposed patients within the analysis, which may have implications for the interpretability of the mucosal findings.

It is becoming clear that endoscopic predictors of $H$. pylori status exist, and when used appropriately can contribute to accurate diagnosis by the general 
endoscopist. We suggest that a close white-light examination of the stomach should be attempted in patients with symptoms of dyspepsia and no known previous history of $\mathrm{H}$. pylori, to attempt to identify the RAC. Our study also suggests that further examination under NBI may identify patients with RAC present, which was not appreciated under WLE. Presence of the RAC should be a reliable sign that $H$. pylori is not present and may avoid the need for gastric biopsies. If the RAC cannot be identified, the presence of diffuse gastric redness or mucosal oedema suggest the possibility of $\mathrm{H}$. pylori infection and may warrant biopsy, stool antigen or breath testing. In the absence of any of these features, if map-like redness is present then an $H$. pylori eradicated status can be suspected, and consideration of future surveillance may be justified based on the patient risk of gastric cancer. Other aids to assessment of these features could include mucolytic agents to ensure good mucosal views ${ }^{33}$ and we suggest their presence or absence could be included in the gastroscopy report for all patients.

\section{CONCLUSIONS}

This study introduces a simple algorithm for classification of $H$. pylori status that may be applied during endoscopy and acts as a decision aid for further diagnostic tests or actions. This algorithm has been prospectively validated in a small sample of patients, in a single centre.

We propose that further research in this area includes a larger patient cohort for prospective validation of this approach. This validation should ideally be conducted in a range of centres with endoscopes from varied manufacturers and undertaken by endoscopists with a range of experience. It will also be beneficial to evaluate the learning curve and performance curve for adoption of this procedure, and to assess interoperator variability.

Contributors NP planned and supervised the study. BG and NP conducted data collection and analysis. JT, BG and NP interpreted and analysed the data. BG prepared the report for submission; NP and JT reviewed and approved the submission. BG submitted the report and is responsible for the overall content as guarantor.

Funding The authors have not declared a specific grant for this research from any funding agency in the public, commercial or not-for-profit sectors.

Competing interests None declared.

Patient consent for publication Not required.

Ethics approval The study was registered with the local research ethics committee, No. 14/L0/1525.

Provenance and peer review Not commissioned; externally peer reviewed.

Data availability statement Data are available on reasonable request. The data shall be available on reasonable request from contacting the corresponding author on bglover@imperial.ac.uk.

Supplemental material This content has been supplied by the author(s). It has not been vetted by BMJ Publishing Group Limited (BMJ) and may not have been peer-reviewed. Any opinions or recommendations discussed are solely those of the author(s) and are not endorsed by BMJ. BMJ disclaims all liability and responsibility arising from any reliance placed on the content. Where the content includes any translated material, BMJ does not warrant the accuracy and reliability of the translations (including but not limited to local regulations, clinical guidelines, terminology, drug names and drug dosages), and is not responsible for any error and/or omissions arising from translation and adaptation or otherwise.
Open access This is an open access article distributed in accordance with the Creative Commons Attribution Non Commercial (CC BY-NC 4.0) license, which permits others to distribute, remix, adapt, build upon this work non-commercially, and license their derivative works on different terms, provided the original work is properly cited, appropriate credit is given, any changes made indicated, and the use is non-commercial. See: http://creativecommons.org/licenses/by-nc/4.0/.

ORCID iD

Ben Glover http://orcid.org/0000-0003-3043-0012

\section{REFERENCES}

1 Correa P. A human model of gastric carcinogenesis. Cancer Res 1988;48:3554-60.

2 Sugano K, Tack J, Kuipers EJ, et al. Kyoto global consensus report on Helicobacter pylori gastritis. Gut 2015;64:1353-67.

3 Leung WK, Wong IOL, Cheung KS, et al. Effects of Helicobacter pylori treatment on incidence of gastric cancer in older individuals. Gastroenterology 2018;155:67-75.

4 Sabbagh P, Mohammadnia-Afrouzi M, Javanian M, et al. Diagnostic methods for Helicobacter pylori infection: ideals, options, and limitations. Eur J Clin Microbiol Infect Dis 2019;38:55-66.

5 Lee JWJ, Lim LG, Yeoh KG. Advanced endoscopic imaging in gastric neoplasia and preneoplasia. BMJ Open Gastroenterol 2017;4:e000105

6 Kato T, Yagi N, Kamada T, et al. Diagnosis of Helicobacter pylori infection in gastric mucosa by endoscopic features: a multicenter prospective study. Dig Endosc 2013;25:508-18.

7 Yagi K, Nakamura A, Sekine A. Characteristic endoscopic and magnified endoscopic findings in the normal stomach without Helicobacter pylori infection. J Gastroenterol Hepatol 2002;17:39-45.

8 Machado RS, Viriato A, Kawakami E, et al. The regular arrangement of collecting venules pattern evaluated by standard endoscope and the absence of antrum nodularity are highly indicative of Helicobacter pylori uninfected gastric mucosa. Dig Liver Dis 2008;40:68-72.

9 Cho J-H, Chang YW, Jang JY, et al. Close observation of gastric mucosal pattern by standard endoscopy can predict Helicobacter pylori infection status. J Gastroenterol Hepatol 2013;28:279-84.

10 Watanabe K, Nagata N, Nakashima R, et al. Predictive findings for Helicobacter pylori-uninfected, -infected and -eradicated gastric mucosa: validation study. World J Gastroenterol 2013;19:4374-9.

11 Garcés-Durán R, García-Rodríguez A, Córdova H, et al. Association between a regular arrangement of collecting venules and absence of Helicobacter pylori infection in a European population. Gastrointest Endosc 2019;90:461-6.

12 Sharma V, Saini S, Saini S, et al. High efficacy of I-scan endoscopy in the diagnosis of Helicobacter pylori infection. Clinical Gastroenterology and Hepatology 2017;15:154.

13 Ebigbo A, Marienhagen J, Messmann $\mathrm{H}$. Regular arrangement of collecting venules and the Kimura-Takemoto classification for the endoscopic diagnosis of Helicobacter pylori infection: evaluation in a Western setting. Dig Endosc 2021;33:587-91.

14 Katake Y, Ichikawa K, Fujio C, et al. Irregular arrangement of collecting venules (IRAC) provides a critical endoscopic insight in Helicobacter pylori-induced gastritis: A secondary publication. Biomed Rep 2013;1:23-7.

15 Alaboudy A, Elbahrawy A, Matsumoto S, et al. Regular arrangement of collecting venules: does patient age affect its accuracy? World $J$ Gastrointest Endosc 2011;3:118-23.

16 Hooi JKY, Lai WY, Ng WK, et al. Global prevalence of Helicobacter pylori infection: systematic review and meta-analysis. Gastroenterology 2017;153:420-9.

17 Gonen C, Simsek I, Sarioglu S, et al. Comparison of high resolution magnifying endoscopy and standard videoendoscopy for the diagnosis of Helicobacter pylori gastritis in routine clinical practice: a prospective study. Helicobacter 2009;14:12-21.

18 Anagnostopoulos GK, Yao K, Kaye P, et al. High-Resolution magnification endoscopy can reliably identify normal gastric mucosa, Helicobacter pylori-associated gastritis, and gastric atrophy. Endoscopy 2007;39:202-7.

19 Pimentel-Nunes P, Libânio D, Lage J, et al. A multicenter prospective study of the real-time use of narrow-band imaging in the diagnosis of premalignant gastric conditions and lesions. Endoscopy 2016;48:723-30.

20 Hussain I, Ang TL. Evidence based review of the impact of image enhanced endoscopy in the diagnosis of gastric disorders. World $\mathrm{J}$ Gastrointest Endosc 2016;8:741-55. 
21 Gomes A, Skare TL, Prestes MA, et al. Conventional videoendoscopy can identify Helicobacter pylori gastritis? Arq Bras Cir Dig 2016;29:73-6.

22 Yan S-L, Wu S-T, Chen C-H, et al. Mucosal patterns of Helicobacter pylori-related gastritis without atrophy in the gastric corpus using standard endoscopy. World J Gastroenterol 2010;16:496-500.

23 Nishikawa Y, Ikeda Y, Murakami H, et al. Classification of atrophic mucosal patterns on blue laser imaging for endoscopic diagnosis of Helicobacter pylori-related gastritis: a retrospective, observational study. PLoS One 2018;13:e0193197.

24 Yagi K, Saka A, Nozawa Y, et al. Prediction of Helicobacter pylori status by conventional endoscopy, narrow-band imaging magnifying endoscopy in stomach after endoscopic resection of gastric cancer. Helicobacter 2014;19:111-5.

25 Yagi K, Aruga Y, Nakamura A, et al. Regular arrangement of collecting venules (RAC): a characteristic endoscopic feature of Helicobacter pylori-negative normal stomach and its relationship with esophago-gastric adenocarcinoma. J Gastroenterol 2005;40:443-52.

26 Mao T, Wang Y, Yin F, et al. Association of endoscopic features of gastric mucosa with Helicobacter pylori infection in chinese patients. Gastroenterol Res Pract 2016;2016:1-7.

27 Gupta S, Li D, El Serag HB, et al. AGA clinical practice guidelines on management of gastric intestinal metaplasia. Gastroenterology 2020;158:693-702.
28 Yoshii S, Mabe K, Watano K, et al. Validity of endoscopic features for the diagnosis of Helicobacter pylori infection status based on the Kyoto classification of gastritis. Dig Endosc 2020;32:74-83.

29 Toyoshima O, Nishizawa T, Sakitani K, et al. Helicobacter pylori eradication improved the Kyoto classification score on endoscopy. JGH Open 2020;4:909-14.

30 Inui M, Ohwada S, Inui Y, et al. Evaluating the accuracy of the endoscopic ABC classification system in diagnosing Helicobacter pylori-infected gastritis. Digestion 2020;101:1-10.

31 Buderer NM. Statistical methodology: I. incorporating the prevalence of disease into the sample size calculation for sensitivity and specificity. Acad Emerg Med 1996;3:895-900.

32 Toyoshima O, Nishizawa T, Koike K. Endoscopic Kyoto classification of Helicobacter pylori infection and gastric cancer risk diagnosis. World J Gastroenterol 2020;26:466-77.

33 Beg S, Ragunath K, Wyman A, et al. Quality Standards in upper gastrointestinal endoscopy: a position statement of the British Society of gastroenterology (Bsg) and association of upper gastrointestinal surgeons of great britain and ireland (AUGIS). Gut 2017;66:1886-99.

$34 \mathrm{Kim}$ DB, Chung WC. Accuracy of endoscopic diagnosis of mild atrophic gastritis with Helicobacter pylori infection. Clin Endosc 2018;51:310-2. 